\title{
BJO
}

\section{Intraoperative extraocular Indocyanine Green (IE-ICG) dye test: a new method of detecting a peeled internal limiting membrane}

K H Park, J-M Hwang, J H Kim, et al.

Br J Ophthalmol 2008 92: 369-372 originally published online January 22, 2008

doi: 10.1136/bjo.2007.125401

Updated information and services can be found at:

http://bjo.bmj.com/content/92/3/369.full.html

Topic collections Articles on similar topics can be found in the following collections

Retina (1005 articles)

Notes

To order reprints of this article go to:

http://bjo.bmj.com/cgi/reprintform

To subscribe to British Journal of Ophthalmology go to:

http://bjo.bmj.com/subscriptions 


\title{
Intraoperative extraocular Indocyanine Green (IE-ICG) dye test: a new method of detecting a peeled internal limiting membrane
}

\author{
K H Park, ${ }^{1,2,3}$ J-M Hwang, ${ }^{1,2,3}$ J H Kim, ${ }^{1,2}$ H G Yu, ${ }^{1,2}$ Y S Yu, ${ }^{1,2}$ H Chung ${ }^{1,2}$
}

${ }^{1}$ Department of Ophthalmology,

College of Medicine, Seoul

National University, Seoul,

Korea; ${ }^{2}$ Seoul Artificial Eye

Center, Seoul National University

Hospital Clinical Research

Institute, Seoul, Korea;

${ }^{3}$ Department of Ophthalmology,

Seoul National University

Bundang Hospital, Seongnam,

Korea

Correspondence to:

$\mathrm{H}$ Chung, Department of

Ophthalmology, College of

Medicine, Seoul National

University, 28 Yongon-dong,

Chongro-gu, Seoul 463-707,

Korea; chungh@sun.ac.kr

Accepted 30 June 2007

Published Online First

22 January 2008

\section{ABSTRACT \\ Aims: To develop an intraoperative, extraocular Indocyanine Green dye staining test (IE-ICG) for the differentiation of a peeled ILM from a thin epiretinal membrane, and to evaluate its efficacy.}

Methods: This was a consecutive observational case and laboratory observational series. We performed ILM peeling in patients with an idiopathic macular hole $(\mathrm{MH}, \mathrm{n}=10)$ and diabetic macular oedema (DME, $n=10$ ) without vital dye staining such as ICG or Trypan Blue. We also performed membrane peeling in patients with an idiopathic epiretinal membrane (ERM, $n=10$ ). Then, the peeled membranes were stained with ICG $(1.25 \mathrm{mg} / \mathrm{ml})$ beyond the operation field and examined under a light microscope. After this examination, membranes were fixed with glutaraldehyde, and an electron microscope was used to confirm whether they were ILMs or thin ERM. The concordance rates between surgeon's intraoperative impression of membranes (SI), IE-ICG results (IT) and histological findings (HF) of peeled membranes were evaluated to reveal the efficacy of IE-ICG. Results: The ILMs were homogenously stained with ICG dye (posivite IE-ICG), and the ERMs were not stained at all by ICG dye (negative IE-ICG). The concordance rate between IT and $\mathrm{HF}$ was $100 \%$ in all three groups of patients. However, concordance rates between SI and IT were 100\% in MH, $80 \%$ in DME and $50 \%$ in ERM, respectively. The surgeon's impression of the membrane is inaccurate, especially in patients with idiopathic epiretinal membrane.

Conclusion: Considering the cost, difficulties of tissue preparation, and the time-consuming process of histological confirmation of an ILM, IE-ICG may be a useful alternative for the differentiation of a peeled ILM and a thin ERM.

Though definite effects remain controversial, many surgeons are performing internal limiting membrane (ILM) peeling to improve surgical outcome in patients with an idiopathic macular hole, diabetic macular oedema or idiopathic epiretinal membrane. ${ }^{1-}$ ${ }^{6}$ ILM peeling is a challenging procedure due to its inborn thinness and transparency. To improve its visibility, a vital dye such as Indocyanine Green, or Trypan Blue has been used for facilitating ILM peeling. ${ }^{7-11}$ However, many reports suggest that vital dyes are possibly toxic when applied during macular surgeries. ${ }^{12-23}$ Recently, intravitreal triamcinolone acetonide (IVTA) was used as an alternative option of vital dye for ILM peeling, ${ }^{24}$ while some other expert surgeons have performed ILM peeling without any enhancing materials such as ICG, Trypan Blue, or IVTA. ${ }^{18}{ }^{25}$ In cases when ILM peeling is conducted with triamcinolone or without a vital dye, it is difficult to know whether the peeled membrane is the ILM or the thin epiretinal membrane. Surgeons usually depend on a personal feeling or impression during the peeling procedure, or use some other supportive sign such as petechial haemorrhage on the macular surface, disappearance of light reflex on the macular area or the scrolling nature of the peeled membrane. However, the only way to confirm that a peeled membrane as the ILM is to perform a histological examination, but such a confirmation involves high costs and is a difficult, time-consuming procedure. Further, the histological examination requires fixation and embedding, and would not be available until after completion of the case. Thus, we have developed a new method, which we refer to as the intraoperative extraocular ICG dye test (IE-ICG), which provides a feasible alternative for differentiating the ILM from the thin epiretinal membrane. Moreover, the devised method is non-toxic, rapid and cheap. We also evaluated the efficacy of the IE-ICG in patients with an idiopathic macular hole, diabetic macular oedema or an idiopathic epiretinal membrane.

\section{MATERIALS AND METHODS}

\section{Patients}

Patients with an idiopathic macular hole $(\mathrm{n}=10)$, diabetic macular oedema $(n=10)$ and idiopathic epiretinal membrane $(n=10)$ were enrolled in this study. All operations were performed by one experienced vitreoretinal surgeon (KHP). The surgical procedure was briefly as follows. In macular hole patients, standard three-port pars planar vitrectomy was performed. Posterior vitreous detachment (PVD) was induced with active suction of ocutome over the optic disc if the PVD is not already present. ILM peeling was performed using end-gripping Eckardt forceps using an intravitreal injection of triamcinolone to enhance ILM visibility in five patients. The other five patients received ILM peeling without any enhancing material-for example, ICG, Trypan Blue or triamcinolone acetonide. The presumed ILM was removed from the eye and the IE-ICG performed off the surgical field.

In diabetic macular oedema patients, vitrectomy was performed, and PVD was induced if not present. ILM peeling was performed as described above. In five patients, triamcinolone was used, and in the other five no enhancing material was used.

In patients with idiopathic ERM, a vitrectomy was performed using the procedure described above, and ERM peeling was performed using end-gripping Eckardt forceps. ILM peeling was not attempted. An IE-ICG dye test was performed to determine whether the membrane was the ERM or ILM. 


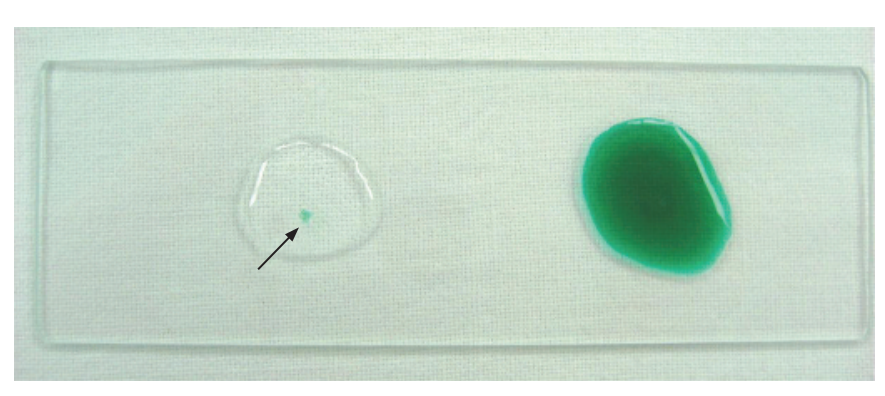

Figure 1 Intraoperative extraocular ICG (IE-ICG) dye test. The peeled membrane was dipped into a drop of ICG dye $(1.25 \mathrm{mg} / \mathrm{ml})$ on a slide glass for $30 \mathrm{~s}$ (right side of slide glass). The membrane was then suspended in a drop of BSS for examination under a light microscope (left side of the slide glass). A tiny ICG stained membrane was visible in a drop of BSS (arrow). If the peeled membrane is an ILM, the membrane is homogenously stained by the green colour of the ICG dye (IE-ICG positive, fig 2A).

\section{Intraoperative extraocular ICG dye test (IE-ICG)}

The presumed ILM or ERM was removed as one piece from the eye and suspended in balanced salt solution (BSS). It was then dipped into a drop of ICG dye $(1.25 \mathrm{mg} / \mathrm{ml})$ on a slide glass for $30 \mathrm{~s}$. Care must be taken to unfold the membrane with 30gauge needle, if it is folded. This might improve exposure of membrane to the ICG dye and reduce the false negative results. The membrane was then resuspended in BSS and gently washed for $30 \mathrm{~s}$. It was then suspended in a drop of BSS on a glass slide for examination under a light microscope (fig 1). When the peeled membrane was an ILM, the membrane was homogenously stained with a green colour due to ICG dye (IE-ICG positive; fig 2A). On the other hand, if it was an ERM, the membrane was not stained at all (IE-ICG negative, fig 2C).

Two experienced readers $(\mathrm{PKH}$ and $\mathrm{KJH})$, masked to the subject information, interpreted the results of IE-ICG. Interand intraobserver variability was analysed by the $\kappa$ statistic. $^{26}$ There was no intra-observer variability in the interpretation of IE-ICG. Inter-observer variability was observed only in the interpretation of membrane from ERM patients, and the $\kappa$ value was 0.80 . We also calculated the concordance rate between the surgeon's impression and IE-ICG results, and between the IEICG and histological findings in each group of patients.

\section{Histological examination}

After the IE-ICG, the peeled membranes were processed for histological examination to confirm their natures. Specimens were fixed in $4 \%$ phosphate-buffered glutaraldehyde solution and postfixed in osmium tetroxide. After dehydration in graded concentration of ethanol, membranes were embedded in Epon 812. For light-microscopic examination, a semithin section of $400 \mathrm{~nm}$ was stained with Toluidine Blue. An ultrathin section of $60 \mathrm{~nm}$ was contrasted with uranyl acetate and lead citrate for electron microscopy. Analysis and imaging were performed with an Olympus BX 51 light microscope (Olympus, Tokyo) and a Hitachi 7100 electron microscope (Hitachi, Tokyo).

\section{RESULTS}

All 30 patients were tested using the IE-ICG successfully without tissue loss. ICG dye mixed with distilled water had not shown any storage-related problems in performing the IE-ICG test until 1 month after make-up. We did not have any further
Figure 2 (A) Light-microscopic findings demonstrating positive IE-ICG dye test results $(\times 40)$. If the peeled membrane is the ILM, the membrane is homogenously stained with a green colour due to the ICG dye ("+" by the IE-ICG dye test). (B) Electron-microscopic findings of the same membrane showing a homogenous structure without a cellular component, proving that the membrane is in fact an ILM $(\times 4000$, bar $=8.75 \mu \mathrm{m})$. (C) Light microscopic finding demonstrates negative results by the IE-ICG dye test $(\times 40)$. If the membrane is an ERM, it is not stained by ICG dye ("-" IE-ICG dye test). (D) Electron-microscopic finding of the same membrane showing irregularly intermingled collagen and attached cells adjacent to the membrane proving that the membrane is an ERM $(\times 7000$, bar $=5.0 \mu \mathrm{m}$ ). (E) Light-microscopic finding of an ERM obtained by epiretinal membrane peeling $(\times 40)$. Removed membranes were observed to have two components; one was not stained with ICG dye (indicating that it was an ERM), while the other was stained by ICG dye (arrow) indicating that it was ILM. (F) Electron-microscopic findings of the same membrane. The two components of the adherent membrane were resolved. The upper part was an ERM (arrowhead) and the lower more homogenous membrane an ILM (arrow) $(\times 9000$, bar $=3.89 \mu \mathrm{m})$.
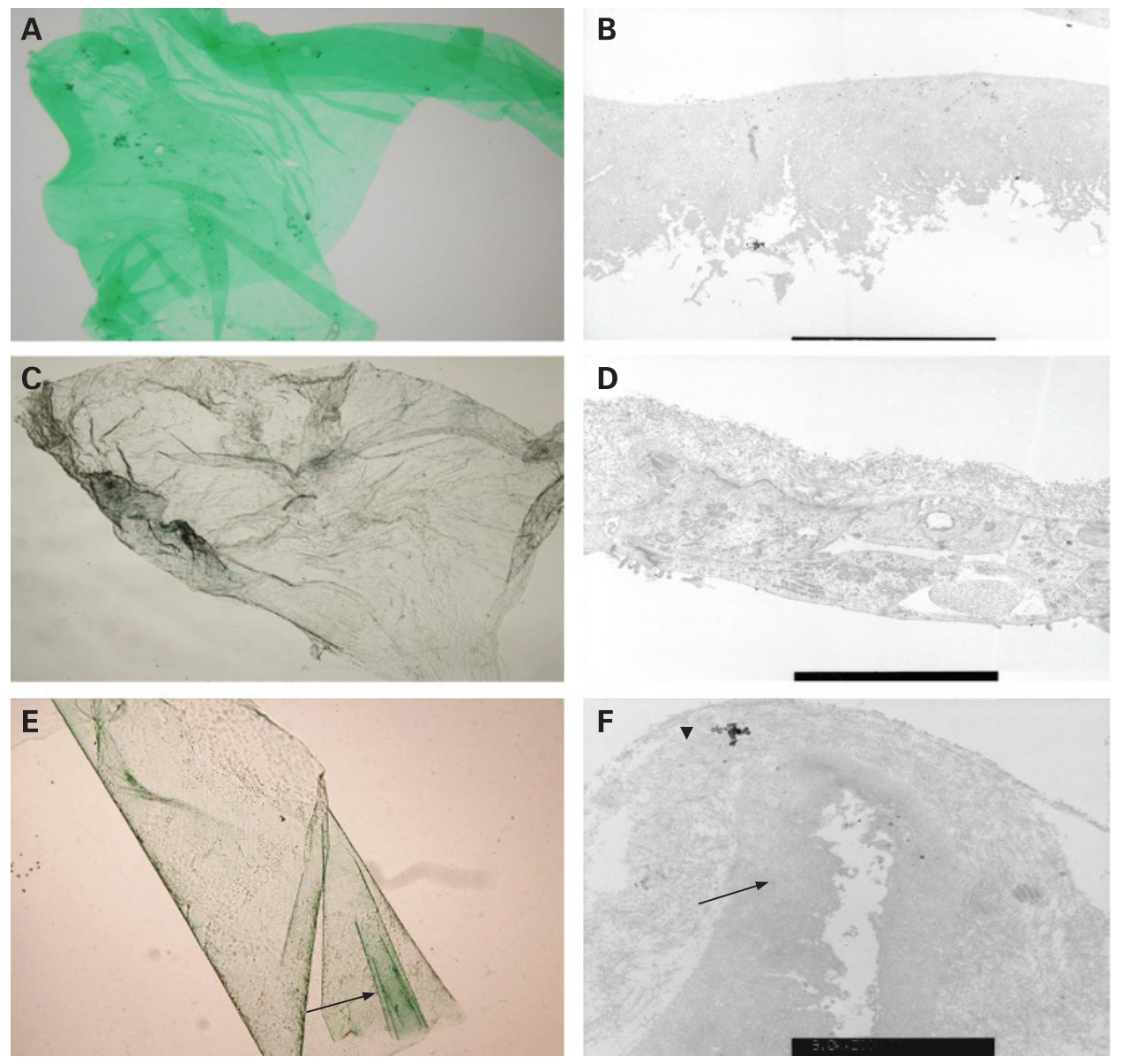
Table 1 Demographics, visual outcome and test results of the patients suffering from an idiopathic macular hole, diabetic macular oedema or idiopathic epiretinal membrane

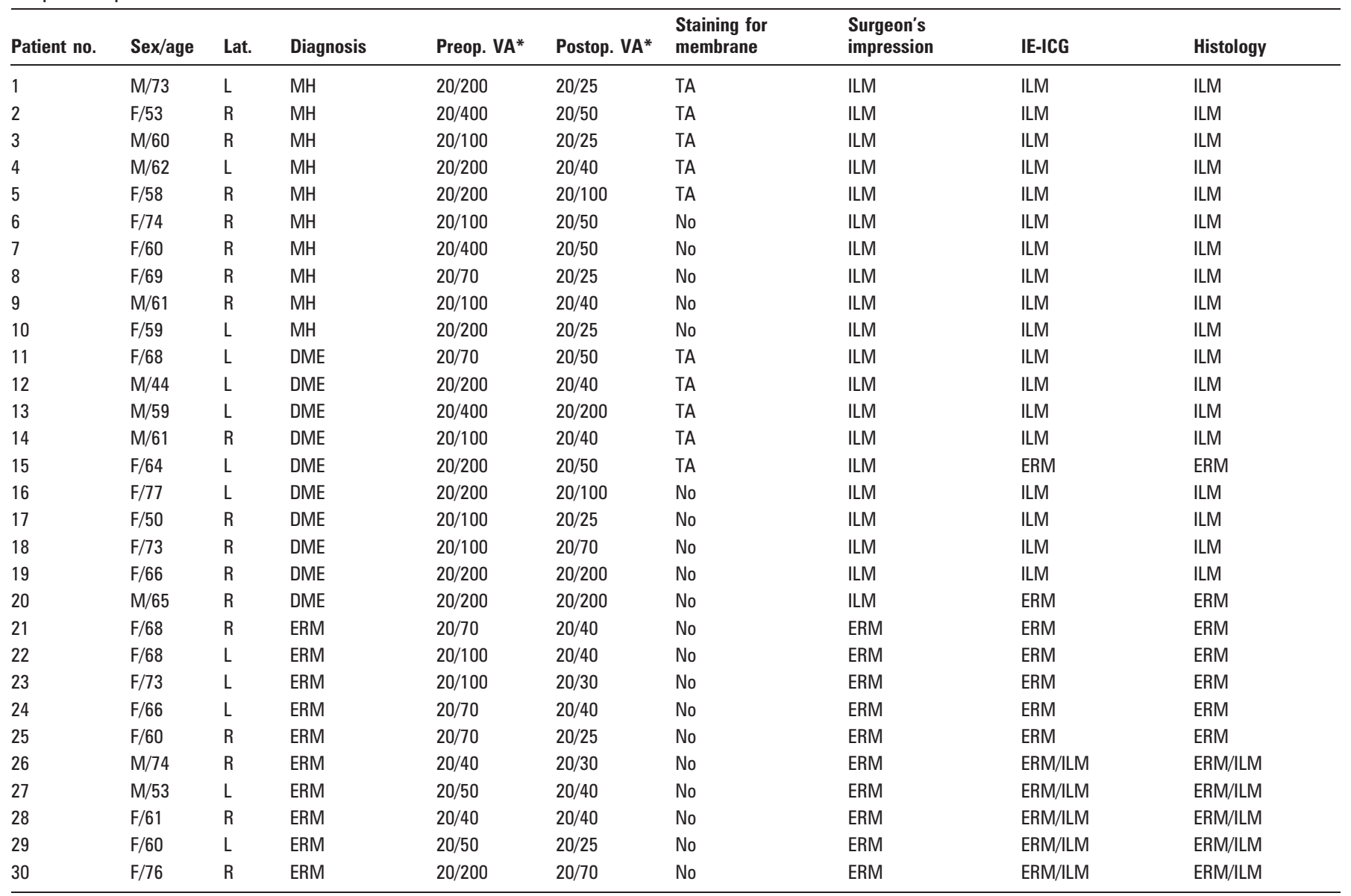

*Visual acuity was measured using a Snellen chart.

DME, diabetic macular oedema; ERM, epiretinal membrane; F, female; ILM, internal limiting membrane; L, left eye; M, male; MH, idiopathic macular hole; No, no enhancing materia for ILM peeling; R, right eye; TA, intravitreal triamcinolone acetonide for ILM peeling.

information concerning the longevity of ICG dye in storage, because that was not the aim of this study.

Ten of the 10 patients (100\%) who underwent macular hole surgery with ILM peeling had a positive IE-ICG. It showed homogeneous greenish staining of membrane with ICG dye and was confirmed from the EM study as ILM (fig 2A, B). Eight of the 10 patients with diabetic macular oedema (80\%) who underwent vitrectomy with ILM peeling had a positive IE-ICG. The other two presumed ILMs were negative by the IE-ICG, indicating that they were not ILMs but rather thin posterior hyaloid membranes (fig 2C) and confirmed as ERM by histological study (fig 2D). We carried out an additional ILM peeling procedure with the triamcinolone acetonide. Five of the 10 patients with idiopathic ERM who underwent ERM peeling also had a negative IE-ICG, and the other five had a mixed positive and negative, indicating that the ILM was inadvertently peeled during the membrane peeling procedure (fig $2 \mathrm{E}, \mathrm{F}$ ). In ERM patients, we did not perform a further procedure because we did not intend to peel the ILM. The concordance rates between the surgeon's impression and the IE-ICG results were $100 \%$ for macular hole surgery, $80 \%$ for diabetic macular oedema surgery and $50 \%$ for ERM surgery. However, the concordance rate between the IE-ICG and histological examination was $100 \%$ in all three groups (table 1).

These results showed that a surgeon's impressions related to ILM and ERM peeling are inaccurate in diabetic macular oedema and ERM patients. They also suggest that IE-ICG is as accurate as histological examination in terms of discriminating ILM from ERM.

\section{DISCUSSION}

Considerations of the possible toxicity of vital dye during the ILM peeling procedure have encouraged some surgeons to perform ILM peeling without vital dye staining or with the use of intravitreal triamcinolone. ${ }^{18}{ }^{25}$ In these situations, surgeons depend on their own feelings and impressions to determine the nature of a peeled membrane. However, this method is quite subjective and always has the possibility of introducing substantial errors. Actually, our findings revealed that a surgeon's impressions during the surgical procedure are likely to be accurate in patients with macular hole, which means IEICG may be unnecessary in patients with an idiopathic macular hole. However, more complicated cases such as a macular hole with retinal detachment in patients with high myopia showed a multiplicity of epiretinal components of peeled membrane. ${ }^{27}$ The IE-ICG may be helpful in revealing the nature of a peeled membrane in these more complicated cases of macular hole patients. The surgeon's impression is somewhat inaccurate in patients with diabetic macular oedema, whereas in patients with an epiretinal membrane, the present study shows that there is a $50 \%$ chance of misdiagnosing the peeled membrane, which demonstrates that a surgeon's impression of the nature of a peeled membrane is inaccurate in patients with diabetic 
macular oedema and an epiretinal membrane. Thus, if surgeons want to guarantee an ILM peeling in these patients, they should use vital dye or some other method utilised to determine whether a peeled membrane is in fact the ILM.

Histological confirmation is currently the only method capable of confirming the nature of a peeled membrane, but it is not a straightforward method and almost impossible for every peeled membrane. Therefore, a more feasible, less expensive method that can reveal the nature of a peeled membrane and be performed during the surgical procedure is required. It was already known that ICG selectively stains the internal limiting membrane. ${ }^{28}$ However, the usage of ICG as adjunctive during surgical procedures is limited to intraocular injections intended to enhance ILM visibility. As yet, no one has attempted ICG staining of a peeled membrane outside the eyeball as a ready means of identifying the membrane type.

Therefore, we developed a new feasible method for differentiating the ILM from thin epiretinal membrane by dying removed membrane outside the operation field with ICG during operations. The described IE-ICG needs only a few minutes to produce a definitive result and can be performed in parallel with the surgical procedure, and so it may provide information as to whether an additional procedure is necessary or not. The devised test is cheap - it requires only a drop of ICG dye - and it does not require processes like fixation, embedding or tissue sectioning. Moreover, it is free of toxicity concerns because all procedures are performed outside the operation fields. In addition to these advantages, its sensitivity and specificity were found to be $100 \%$ versus histological findings irrespective of the disease groups. This means that the devised test is both feasible and highly accurate.

However, several concerns remain. First is the need for IEICG. The precise nature of the peeled membrane may not be crucial for the results of macular surgeries. One important consideration may not be whether the peeled membrane is ILM or not, but how to peel it to the intended amount while minimising trauma to the macula. Therefore, some may underestimate the necessity of this test. However, in the absence of a test like the IE-ICG, it is difficult to address questions such as, "How do you know the membrane you peeled without vital dye is the ILM?" without resorting to histological results. So, even though the clinical usefulness of IEICG may be limited in retinal surgeons, its academic value is still sufficient to sustain this test as a feasible alternative for histological examination. Second, the concordance rate between surgeons' impressions of membrane peeling without any vital dyes and IE-ICG results may depend on surgeons' experience. As a beginner, the concordance rate may be low, and it may be high in experts. It is possible that the IE-ICG could be used by surgeons to hone their intraoperative impression concerning the natures of the peeled membrane through trial-and-error basis learning. Third, inter-observer variability was observed in interpretation of membrane from ERM patients. However, this discrepancy was eliminated with further education and selflearning gained from the results of histological examination.

In conclusion, we describe a new test, which we refer to as the IE-ICG. Because of its negligible cost, ease of tissue preparation and rapidity versus histological confirmation, we believe that the IE-ICG will be found to be a useful ready alternative for the differentiation of peeled ILMs and thin epiretinal membranes.

Acknowledgements: The authors thank S-y Park and H-s Lee, Department of Pathology of Seoul National University Bundang Hospital, for their help in obtaining and interpreting the EM images of ILM.
Funding: This study was supported by the research grant of Seoul National University Bundang Hospital (02-2006-016).

Competing interests: None declared.

Ethics approval: Institutional Ethics Committee approval was granted for the study.

Patient consent: Informed patient consent was obtained before surgery.

\section{REFERENCES}

1. Mester V, Kuhn F. Internal limiting membrane removal in the management of fullthickness macular holes. Am J Ophthalmol 2000;129:769-77.

2. Brooks HL. Macular hole surgery with and without internal limiting membrane peeling. Ophthalmology 2000;107:1939-49.

3. Cheng L, Azen SP, El-Bradey MH, et al. Effects of preoperative and postoperative epiretinal membranes on macular hole closure and visual restoration. Ophthalmology 2002;109:1514-20.

4. Tognetto D, Grandin R, Sanguinetti G, et al. Internal limiting membrane removal during macular hole surgery. Results of a multicenter retrospective study. Ophthalmology 2006;113:1401-10.

5. Recchia FM, Ruby AJ, Carvalho Recchia CA. Pars plana vitrectomy with removal of the internal limiting membrane in the treatment of persistent diabetic macular edema. Am J Ophthalmol 2005;139:447-54.

6. Yanyali A, Nohutcu AR, Horoxoqlu F, et al. Modified grid laser photocoagulation versus pars plana vitrectomy with internal imiting membrane removal in diabetic macular edema. Am J Ophthalmol 2005;139:795-801.

7. Kadonosono K, Itoh N, Uchio E, et al. Staining of internal limiting membrane in macular hole surgery. Arch Ophthalmol 2000;118:1116-8.

8. Burk SE, Da Mata AP, Snyder ME, et al. Indocyanine Green-assisted peeling of the retinal internal limiting membrane. Ophthalmology 2000;107:2010-4.

9. Ando R, Sasano K, Ohba N, et al. Anatomic and visual outcomes after Indocyanine Green-assisted peeling of the retinal internal limiting membrane in idiopathic macular hole surgery. Am J Ophthalmol 2004;137:609-14.

10. Li K, Wong D, Hiscott $P$, et al. Trypan Blue staining of internal limiting membrane and epiretinal membrane during vitrectomy: visual results and histopathological findings. Br J Ophthalmol 2003;87:216-9.

11. Teba FA, Mohr A, Eckardt C, et al. Trypan Blue staining in vitreoretinal surgery. Ophthalmology 2003;110:2409-12.

12. Tadayoni R, Paques M, Girmens JF, et al. Persistence of fundus fluorescence after use of Indocyanine Green for macular surgery. Ophthalmology 2003;110:604-8.

13. Gandorfer A, Haritoglou C, Gandorfer A, et al. Retinal damage from Indocyanine Green in experimental macular surgery. Invest Ophthalmol Vis Sci 2003;44:316-23.

14. Sippy BD, Engelbrecht NE, Hubbard GB, et al. Indocyanine green effect on cultured human retinal pigment epithelial cells: implication for macular hole surgery. Am J Ophthalmol 2001;132:433-5.

15. Engelbrecht NE, Freeman J, Sternberg P Jr, et al. Retinal pigment epithelial changes after macular hole surgery with indocyanine green-assisted internal limiting membrane peeling. Am J Ophthalmol 2002;133:89-94.

16. Uemoto R. Yamamoto S, Takeuchi S. Changes in retinal pigment epithelium after indocyanine green-assisted internal limiting lamina peeling during macular hole surgery. Am J Ophthalmol 2005;140:752-5.

17. Ikagawa $\mathbf{H}$, Yoneda $\mathbf{M}$, Iwaki $\mathbf{M}$, et al. Chemical toxicity of indocyanine green damages retinal pigment epithelium. Invest Ophthalmol Vis Sci 2005;46:2531-9.

18. Schumann RG, Schaumberger MM, Rohleder M, et al. Ultrastructure of the vitreomacular interface in full-thickness idiopathic macular holes: a consecutive analysis of 100 cases. Am J Ophthalmol 2006;141:1112-9.

19. Gass CA, Haritoglou C, Schauberger M, et al. Functional outcome of macular hole surgery with and without indocyanine green-assisted peeling of the internal limiting membrane. Graefes Arch Clin Exp Ophthalmol 2003;241:716-20.

20. Kanda S, Uemura A, Yamashita T, et al. Visual field defects after intravitreous administration of Indocyanine Green in macular hole surgery. Arch Ophthalmol 2004;122:1447-51.

21. Narayanan R, Kenney MC, Kamjoo S, et al. Trypan Blue: effect on retinal pigment epithelial and neurosensory retinal cells. Invest Ophthalmol Vis Sci 2005;46:304-9.

22. Rezai KA, Farrokh-Siar L, Gasyna EM, et al. Trypan Blue induces apoptosis in human retinal pigment epithelial cells. Am J Ophthalmol 2004;138:492-5.

23. Lüke C, Lüke M, Dietlein TS, et al. Retinal tolerance to dyes. $\mathrm{Br} J$ Ophthalmol 2005;89:1188-91.

24. Kimura H, Kuroda S, Nagata M. Triamcinolone acetonide-assisted peeling of the internal limiting membrane. Am J Ophthalmol 2004;137:172-3.

25. Haritoglou C, Reiniger IW, Schaumberger M, et al. Five-year follow-up of macular hole surgery with peeling of the internal limiting membrane. Update of a prospective study. Retina 2006;26:618-22.

26. SAS Institute. SAS/STAT user's guide. Ver. 8, vol. 2.Cary: SAS Institute, 1989:1247-2552.

27. Sakaguchi H, lkuno $Y$, Choi J, et al. Multiple components of epiretinal tissues detected by triamcinolone and Indocyanine Green in macular hole and retinal detachment as a result of high myopia. Am J Ophthalmol 2004;138:1079-81.

28. Gandorfer A, Messmer EM, Ulbig MW, et al. Indocyanine Green selectively stains the internal limiting membrane. Am J Ophthalmol 2001;131:387-8. 